\title{
Rifaximin and Crohn's Disease: A New Solution to an Old Problem?
}

\author{
A. S. Day $\cdot$ R. B. Gearry
}

Published online: 13 February 2010

(C) Springer Science+Business Media, LLC 2010

\section{Introduction}

It is clear that the intestinal flora, and interactions between these flora and the host, contribute strongly to the pathogenesis of Crohn's disease (CD). Modification of the intestinal flora by various modalities has been explored in animal models of gut inflammation and in the human setting. In this issue, Shafran and Burgunder [1] illustrate the outcomes of the use of rifaximin, a non-absorbable antibiotic, in the setting of $\mathrm{CD}$. This report incorporated the retrospective analysis of rifaximin use in 68 adults with active $\mathrm{CD}$. These individuals were treated with rifaximin for a median of 16.6 weeks, with almost all treated with $600 \mathrm{mg}$ daily. Overall, two-thirds of these patients were noted to enter remission with rifaximin therapy.

\footnotetext{
A. S. Day $(\bowtie)$

Department of Paediatrics, University of Otago,

P.O. Box 4345, Christchurch 8140, New Zealand

e-mail: andrew.day@otago.ac.nz

R. B. Gearry

Department of Medicine, University of Otago,

Christchurch, New Zealand

\section{A. S. Day}

Department of Paediatrics,

Christchurch Hospital, Christchurch, New Zealand

\section{R. B. Gearry}

Department of Gastroenterology, Christchurch Hospital,

Christchurch, New Zealand
}

\section{Intestinal Flora, Antibiotics, and Crohn's Disease}

Currently, the precise aetiology of $\mathrm{CD}$ is unclear. The best accepted hypothesis is of interactions between the intestinal microorganisms (or their by-products) and the gastrointestinal mucosa, with a consequent dysregulated response, in genetically predisposed individuals [2-4].

Many investigators have examined aspects of the intestinal flora over recent years. Studies have focused on various individual organisms: these include Mycobacterium paratuberculosis, Bacteroides vulgatus, Listeria monocytogenes and Escherichia coli subtypes amongst many others. To date, none of these organisms have been conclusively shown to be implicated in CD. Some recent studies have focused upon the mucous-associated floraorganisms inhabiting the mucous layer adjacent to the intestinal epithelium [5, 6]. Organisms in this niche include the enterohepatic Helicobacters and non-jejuni Campylobacters, such as Campylobacter concisus.

As well as studies examining the potential roles of individual organisms in the pathogenesis of $\mathrm{CD}$, several studies have examined the overall patterns of the flora in $\mathrm{CD}$. These studies have utilised various molecular techniques, such as FISH, to define the balances between particular groups of bacteria in the setting of $\mathrm{CD}$ and in the normal control gut. However, these studies have provided some conflicting results, which may reflect differences in methodologies and in the source of the study material. In a recent study, Swidsinski et al. [7] described the findings of FISH using paraffin-embedded faecal cylinders and demonstrated differences in the biostructure of the flora in $\mathrm{CD}$ compared to controls.

There is much data, from animal models and human observations, supporting the central role of the intestinal microflora in the pathogenetic events of $\mathrm{CD}$. In murine 
models of gut inflammation, mice maintained in conventional conditions develop disease, whereas those housed under germ free conditions do not [8]. Treatment of interleukin (IL)-10 deficient mice with either antibiotics or probiotics leads to prevention or attenuation of the colitis [9, 10]. Furthermore, diversion of the colonic contents from an area of inflammation can lead to resolution or prevent recurrence of disease: these findings are reinforced by the finding of inflammatory changes consequent to the flow of intestinal contents into excluded ileal segments [11]. In addition, various therapies that are shown to or implicated in modification of the flora, are able to improve inflammation in some individuals.

Therapies that modify the intestinal flora include exclusive enteral nutrition [12], probiotics and antibiotics [9, 10]. Animal data supports antibiotics having antiinflammatory effects; studies in patients with CD show some support for metronidazole and ciprofloxacin. Metronidazole has particular roles in preventing the recurrence of disease after surgically induced remission, in perianal disease and may have a role in colonic CD [13]. This drug does not, however, have a role in UC (except for in pouchitis). Although data supports these agents, this data is not strong, and antibiotic use is limited to response and side effects.

It is important to note that while antibiotic therapy has been shown to be effective in some studies, the precise mechanism of action is not understood. At this point in time the microbe(s) implicated in CD pathogenesis have not been identified, and it is not clear whether such microbes are the cause of $\mathrm{CD}$ or simply perpetuate inflammation due to a leaky epithelial barrier. Further investigation of host-microbial interactions is vital to determining which antibiotics are likely to be effective and at what point in the disease process.

\section{Rifaximin and Crohn's Disease}

Rifaximin is a non-systemic antibiotic, since it is essentially non-absorbable from the gut. It has particular indications for traveller's diarrhoea. There has been interest in recent years in the potential roles of rifaximin in CD.

Shafran and Johnson [14] evaluated rifaximin in an open-label study of 29 patients with active CD. These patients with mild to moderate elevated disease activity scores (CDAI) were administered rifaximin $200 \mathrm{mg}$ TID for 4 months and were assessed over time for changes in CDAI scores. On an intention to treat basis, the group had a $43 \%$ fall in CDAI scores over the 4-month period $(P<0.0001)$. Almost $60 \%$ of patients entered remission by the end of the trial period. Side-effects included abdominal pain, fatigue, and headache.
This open-label study has been followed by a PC-RCT involving the comparison of two dosage regimens of Rifaximin to a placebo arm in a total of 83 patients with mildmoderate CD [15]. Overall, the group receiving $800 \mathrm{mg}$ twice daily had less treatment failures and had superior induction of remission compared to once daily administration or placebo (in those with elevated CRP).

A retrospective review of rifaximin in children was published last year [16]. This report incorporated 23 children, with approximately half having $\mathrm{CD}$ and half ulcerative colitis. Overall, $61 \%$ of these children had experienced benefits from rifaximin monotherapy; improvements included resolution of diarrhoea and abdominal pain.

There is also a short report of so-called "ecological niche" therapy, whereby three individuals with active CD were given Rifaximin to induce remission and then maintained with probiotic therapy [17]. This case-based experience suggests an interesting role for antibiotic therapy in the management of CD.

Shafran and Burgunder [1] further extended our understanding of the potential roles of rifaximin in a study, albeit retrospective, which collated the clinical outcomes of 68 patients with $\mathrm{CD}$ who had been treated with rifaximin during a 4-year period. These individuals were prescribed rifaximin for various reasons, including patient reluctance to use immunomodulators or intolerance of immunomodulators. A subset $(n=18)$ had received rifaximin monotherapy and a further group had received corticosteroids along with rifaximin. Overall, around two-thirds of the patients had a response with reduction in PCDAI. The response rates were greater in those who had not received corticosteroids along with rifaximin (but this may reflect a greater severity of disease in the latter group). A further feature of note in this single-centre report is that rifaximin appeared to be helpful in the maintenance of remission in a small group of individuals.

The next steps in this story should be a coordinated and consistent approach to dosing regimens, elucidating the specific groups of patients most likely to respond to rifaximin, and establishing clear biological and clinical outcomes to rifaximin therapy. Such studies are now required to fully and clearly define the roles that this drug may have in the management of CD in both adults and children.

\section{Conclusions}

This study provides retrospective data of the use of rifaximin in a real-life clinical setting and confirms prior case series and clinical trial data. Rifaximin appears to be establishing an indication in the management of CD. Further prospective studies are now required to elucidate the full extent and nature of this role. 


\section{References}

1. Shafran I, Burgunder P. Adjunctive antibiotic therapy with rifaximin may help reduce Crohn's disease activity. Dig Dis Sci. 2010. doi:10.1007/s10620-009-1111-y

2. Griffiths AM. Specificities of inflammatory bowel disease in childhood. Best Pract Res Clin Gastroenterol. 2004;18:509-523.

3. Sartor RB. Enteric microflora in IBD: pathogens or commensals? Inflamm Bowel Dis. 1997;3:230-235.

4. Fiocchi C. Inflammatory bowel disease: etiology and pathogenesis. Gastroenterology. 1998;115:182-205.

5. Man SM, Zhang L, Day AS, Leach S, Mitchell HM. Detection of enterohepatic and gastric Helicobacter species in fecal specimens of children with Crohn's Disease. Helicobacter. 2008;13:234 238.

6. Zhang L, Man SM, Day AS, et al. Non-jejuni Campylobacter species are associated with Crohn's disease in children. J Clin Micro. 2009;47:453-455.

7. Swidsinski A, Loening-Baucke V, Vaneechoutte M, Doerffel Y. Active Crohn's disease and ulcerative colitis can be specifically diagnosed and monitored based on the biostructure of the fecal flora. Inflamm Bowel Dis. 2008;14:147-161.

8. Rath HC, Schultz M, Freitag R, et al. Different subsets of enteric bacteria induce and perpetuate experimental colitis in rats and mice. Infect Immun. 2001;69:2277-2285.

9. Madsen KL, Doyle JS, Tavernini MM, et al. Antibiotic therapy attenuates colitis in interleukin 10 gene-deficient mice. Gastroenterology. 2000;118:1094-1105.
10. Madsen KL, Doyle JS, Jewell LD, Tavernini MM, Fedorak RN. Lactobacillus species prevents colitis in interleukin 10 genedeficient mice. Gastroenterology. 1999;116:1107-1114.

11. D'Haens GR, Geboes K, Peeters M, et al. Early lesions of recurrent Crohn's disease caused by infusion of intestinal contents in excluded ileum. Gastroenterology. 1998;114:262-267.

12. Leach ST, Mitchell HM, Eng WR, Zhang L, Day AS. Sustained modulation of intestinal microflora by exclusive enteral nutrition used to treat children with Crohn's disease. Aliment Pharmacol Ther. 2008;28:724-733.

13. Prantera C, Scribano ML. Antibiotics and probiotics in inflammatory bowel disease: why, when, and how. Curr Opin Gastroenterol. 2009;25:329-333.

14. Shafran I, Johnson LK. An open-label evaluation of rifaximin in the treatment of active Crohn's disease. Curr Med Res Opin. 2005; 1:1165-1169.

15. Prantera C, Lochs H, Campieri M, et al. Antibiotic treatment of Crohn's disease: results of a multicentre, double blind, randomized, placebo-controlled trial with rifaximin. Aliment Pharmacol Ther. 2006;23:1117-1125.

16. Muniyappa P, Gulati R, Mohr F, Hupertz V. Use and safety of rifaximin in children with inflammatory bowel disease. Pediatr Gastroenterol Nutr. 2009;49:400-404.

17. Doman DB, Goldberg HJ, Golding MI. "Ecologic niche" therapy for Crohn's disease with adjunctive rifaximin antibiotic treatment followed by Flora-Q probiotic maintenance therapy. Am J Gastroenterol. 2008;103:251-252. 